Artículo científico

Volumen 31(3):609-617. Septiembre-diciembre, 2020 e-ISSN 2215-3608, doi:10.15517/am.v31i3.39515 http://www.revistas.ucr.ac.cr/index.php/agromeso

\title{
Atrayentes alimenticios en trampas McPhail para captura de mosca de la piña (Diptera: Richardiidae) ${ }^{1}$
}

\section{Food attractants in McPhail traps to capture of pineapple fly (Diptera: Richardiidae)}

\author{
Alfonso Villalobos-Moreno², Jesús Emilio Luque-Z. ${ }^{3}$, Jorge Villamizar-Cobos ${ }^{4}$
}

1 Recepción: 7 de noviembre, 2019. Aceptación: 11 de mayo, 2020. Modificado desde el trabajo de maestría del primer autor: Aportes al conocimiento de la mosca de la piña Melanoloma viatrix Hendel, 1911 (Diptera: Richardiidae). Universidad Nacional de Colombia, Facultad de Agronomía, Colombia.

2 Director Grupo de Investigaciones Entomológicas y Ambientales (GENA). Calle 91 No. 22- 104 Apto 403, Bucaramanga, Colombia. avillalobosmo@unal.edu.co (https://orcid.org/0000-0003-1713-7823).

3 Universidad Nacional de Colombia, Facultad de Ciencias Agropecuarias, Bogotá, Colombia. jluquez@unal.edu.co (https://orcid.org/00000001-6724-9036).

4 Universidad Industrial de Santander, Facultad de Ciencias, Laboratorio de Entomología (Pensionado). Bucaramanga, Colombia. villajor2003@gmail.com (https://orcid.org/0000-0003-4370-4537).

\section{Resumen}

Introducción. La mosca de la piña (Melanoloma viatrix Hendel, 1911) fue reportada por primera vez en Colombia en 1991, y se ha convertido en una plaga limitante en el cultivo de piña en este país. Hay escasa información sobre la especie y se requiere estimar su distribución, fluctuación y densidad, para diseñar estrategias de manejo integrado de plagas. Objetivo. Evaluar atrayentes alimenticios como mecanismo de muestreo de Melanoloma viatrix Hendel. Materiales y métodos. Los ensayos se realizaron en fincas piñeras del municipio de Girón, Santander, Colombia, durante el inicio de la primera temporada de lluvias de 2003. Se evaluaron ocho atrayentes alimenticios dentro de trampas McPhail ubicadas en seis bloques al azar dentro de tres cultivos comerciales de piña de la variedad Perolera. Resultados. Los ensayos con atrayentes alimenticios dentro de trampas McPhail, capturaron más de 13 000 individuos, incluyendo dípteros, lepidópteros, himenópteros, neurópteros, hemípteros y ortópteros, pero solo 138 ejemplares de la especie de estudio. Conclusión. Se observaron diferencias significativas en las capturas de $M$. viatrix, siendo trozos de cáscara, extracto de cáscara y jugo natural de piña, los atrayentes que presentaron los mejores resultados de captura.

Palabras claves: piña Perolera, Melanoloma viatrix, Colombia, muestreo de plagas.

\begin{abstract}
Introduction. The pineapple fly (Melanoloma viatrix Hendel, 1911) was first reported in Colombia in 1991, and has become a limiting pest in pineapple crops in this country. The information about the species is scarce and it is required to estimate its distribution, fluctuation, and density, in order to design integrated pest management strategies. Objective. To evaluate food attractants as a sampling mechanism of Melanoloma viatrix Hendel. Materials and


methods. The tests were carried out in pineapples farms in the municipality of Giron, Santander, Colombia, during the beginning of the first rainy season of 2003. Eight food attractants were evaluated within McPhail traps located in six random blocks within three commercial pineapple crops of the Perolera variety. Results. The McPhail trap food attractant tests captured more than 13000 individuals, including diptera, lepidoptera, hymenoptera, neuroptera, hemiptera, and orthoptera, but only 138 specimens of the study species. Conclusion. Significant differences were observed in the captures of $M$. viatrix, being pineapple rind pieces, pineapple rind extract and natural pineapple juice the attractants that presented the best capture results.

Keywords: Perolera pineapple, Melanoloma viatrix, Colombia, pest sampling.

\section{Introducción}

En 1991 se reportó por primera vez en Colombia el díptero Melanoloma viatrix, perteneciente a la familia Richardiidae (Hendel, 1911), conocido como mosca o gusano de la piña, y que se ha constituido en un grave problema de los cultivos comerciales de piña en los departamentos de Antioquia, Santander, Norte de Santander y Cundinamarca (ICA, 1991; Villalobos-Moreno y Luque, 2012; Villalobos-Moreno et al., 2013). El ataque inicia con la oviposición cerca o en el fruto, luego la larva emergida se introduce y se alimenta de su pulpa formando galerías que facilitan la entrada de microorganismos, lo que reduce la calidad del fruto (Arévalo, 1994; Villalobos-Moreno y Luque, 2012; Villalobos-Moreno et al., 2013).

La piña, especialmente la variedad Perolera, empezó a cultivarse de forma intensiva en Colombia a partir de los años cincuenta, y alcanzó la cúspide de la producción en los años setenta. Sin embargo, a pesar de poseer condiciones agroecológicas ideales para este cultivo, el país nunca ha ocupado un lugar importante en la producción mundial de este cultivo. En este contexto de la economía mundial, varios países centroamericanos y sudamericanos superan a Colombia, tanto en áreas sembradas como en producción, siendo Costa Rica el líder desde hace varios años (Garita, 2014). Históricamente, Colombia ocupa alrededor del puesto once con algo más de 500000 t de piña al año y casi 11000 ha sembradas (FAO, 2012; La República, 2012). Sin embargo, se debe resaltar que ha realizado exportaciones pequeñas y esporádicas tanto de fruta fresca como de productos procesados industrialmente, la mayor parte hacia Venezuela, Estados Unidos, Japón y Europa (Garita, 2014), sin que estos hayan generado mayores impactos en la economía nacional. Costa Rica, Honduras y México compiten, e incluso superan a Colombia, gracias a que han generado proyectos a nivel nacional a los que se suman capitales extranjeros para siembra, producción, procesamiento y exportación de piña fresca y sus derivados (CCI, 2006; Chavarría, 2010; Garita, 2014).

La piña se cultiva con densidades de siembra de 25000 a 50000 plantas por hectárea, en altitudes entre los 330 y los $1400 \mathrm{msnm}$, con temperaturas entre $21^{\circ}$ y $28^{\circ} \mathrm{C}$ y precipitaciones de 1000 a $2500 \mathrm{~mm}$ al año. El suelo es un factor poco limitante para establecer el cultivo, ya que la piña crece en una variada gama de suelos que van desde arenosos hasta arcillosos, pero durante el ciclo fenológico de la planta se requiere una o dos aplicaciones de fertilizante (García y Martínez, 1991; Higuera, 1994; Salazar, 1994; Sandoval y Torres, 2011; Santoyo y Martínez, 2011). Este cultivo tampoco requiere riego suplementario, dado que la planta es resistente a la sequía (Jensen y Salisbury, 1988; García y Martínez, 1991; Higuera, 1994; Salazar, 1994; Sandoval y Torres, 2011; Santoyo y Martínez, 2011).

Dentro de los problemas fitosanitarios del cultivo de la piña, los patógenos están bien controlados con métodos profilácticos o preventivos; se ha comprobado que la inmersión de los colinos durante tres minutos en soluciones con plaguicidas evita el ataque de patógenos (Bonilla, 1992; Galindo, 1994a, b; Aravena, 2005; Sandoval y Torres, 
2011). Sin embargo, se han reportado enfermedades como la pudrición del cogollo (Phytophthora sp.) y la peca o mancha del fruto (Penicillium funiculosum Thom, 1910) (Salazar, 1994; Sandoval y Torres, 2011). Con respecto a las plagas, destacan: sinfílidos (Miriapoda: Symphyla), ácaro rojo (Tetranychus sp.), escama roja circular (Chrysomphalus dictyospermi Morgan, 1889), palomilla de la raíz (Dysmicoccus brevipes Cockerell, 1893), cochinillas (Ceroplastes sp. y Toumeyella sp.), azulina de la piña (Thecla sp.), gusano tornillo de la piña (Castnia sp.), cucarrón del invierno (Podischnus agenor Olivier, 1789) y picudos (Metamasius hebetatus Gyllenhal, 1838 y Rhyncophorus palmarum Linnaeus, 1758) (Gallego y Vélez, 1992; Salazar, 1994; Sandoval y Torres, 2011; Santoyo y Martínez, 2011), así como la mosca de la piña que se reporta como el problema más grave en cultivos comerciales de piña en Colombia (Arévalo y Osorio, 1995). Se han realizado observaciones preliminares sobre biología y hábitos del insecto (Arévalo, 1994; Martínez, 1994; Montilla et al., 2007; Villalobos-Moreno et al., 2009; Villalobos-Moreno y Luque, 2012; Villalobos-Moreno et al., 2013) y dada la escasa información sobre la especie, es necesario profundizar en los estudios básicos, para implementar medidas de manejo más acertadas.

Los métodos de monitoreo de moscas de las frutas más efectivos y ampliamente utilizados, se pueden agrupar en dos clases: alimenticios y sexuales; en los primeros, se usan trampas MacPhail cebadas con diversos tipos de sustancias, y en los segundos, moléculas sexuales muy efectivas en trampas Jackson o sobre esferas de colores (Baker et al., 1985; Katsoyannos et al., 1999; Papadopoulos et al., 2001; Castañera, 2003; Primo-Millo et al., 2003; Heath et al., 2004; Matheus, 2005; OIEA, 2005; Raga y Sato, 2005; ICA, 2011; Gutiérrez et al., 2013; Pacheco, 2016). Las trampas McPhail han sido utilizadas en la detección y control de moscas de las frutas, con muy buenos resultados para Anastrepha spp., utilizando proteína hidrolizada de soya, el jugo de piña y una mezcla de melaza más urea (León, 1988; López, 1988; 1989; Martínez, 1994; Moreno y García, 1994b; Matheus, 2005). Trabajos realizaron por Moreno y García (1994a; 1994b) sobre manejo de la mosca de la piña con evaluación de cebos tóxicos y métodos de trampeo, no han tenido resultados lo suficientemente consistentes como para solucionar la problemática del monitoreo de la plaga.

Sobre la mayor parte de las especies del denominado complejo moscas de las frutas, se tienen un relativo buen conocimiento en aspectos biológicos, ecológicos, control de poblaciones y enemigos naturales, entre otros (Baker et al., 1985; Katsoyannos et al., 1999; Papadopoulos et al., 2001; Castañera, 2003; Primo-Millo et al., 2003; Heath et al., 2004; Matheus, 2005; Raga y Sato, 2005; ICA, 2011; Gutiérrez et al., 2013; Pacheco, 2016), caso contrario a lo que ocurre con M. viatrix (Villalobos-Moreno y Luque, 2012; Villalobos-Moreno et al., 2013). Con respecto al monitoreo poblacional de este complejo, existe una amplia variedad de sustancias hormonales y alimenticias con gran efectividad, e incluso alta especificidad, en la captura de adultos (Heath et al., 2004; OIEA, 2005; Raga y Sato, 2005; Gutiérrez et al., 2013). En el manejo integrado de plagas (MIP), el monitoreo es una actividad esencial y para ello, se utiliza ampliamente proteína hidrolizada en trampas McPhail. Este es un proceso que, debido a los costos, tiene cierta dificultad para ser implementado, por ello, y similar a lo propuesto en la presente investigación, se experimenta con atrayentes artesanales basados en jugos, orina y estiércol (Bateman y Morton, 1981; Mazor et al., 1987; Veloso et al., 1994; Salles, 1997; Rodríguez et al., 2000; Piñero et al., 2003; Heath et al., 2004; Canal et al., 2010).

Debido a la importancia de establecer ciertos parámetros poblacionales para futuros proyectos de manejo integrado como distribución, fluctuación y densidad de Melanoloma viatrix, el objetivo de este trabajo fue evaluar atrayentes alimenticios como mecanismo de muestreo de M. viatrix Hendel. 


\section{Materiales y métodos}

\section{Localización de los ensayos}

La evaluación de los atrayentes alimenticios con trampas McPhail para captura de Melanoloma viatrix, se llevó a cabo según la disponibilidad de permisos para ingresar a las fincas, durante los meses de marzo y abril del 2003, que corresponde al inicio del primer periodo de lluvias del año. Los muestreos se realizaron en fincas de propiedad de los señores Felipe Ayala, Carlos Ruiz y Jorge Fonseca, con cultivos comerciales de piña de la variedad Perolera, ubicados en la vereda Llanadas $\left(7^{\circ} 02^{\prime} 48 \mathrm{~N} ; 7^{\circ} 09^{\prime} 28 \mathrm{O}\right)$ del municipio de Girón, Santander, Colombia (Figura 1), en una altitud promedio de $800 \mathrm{~m}$.

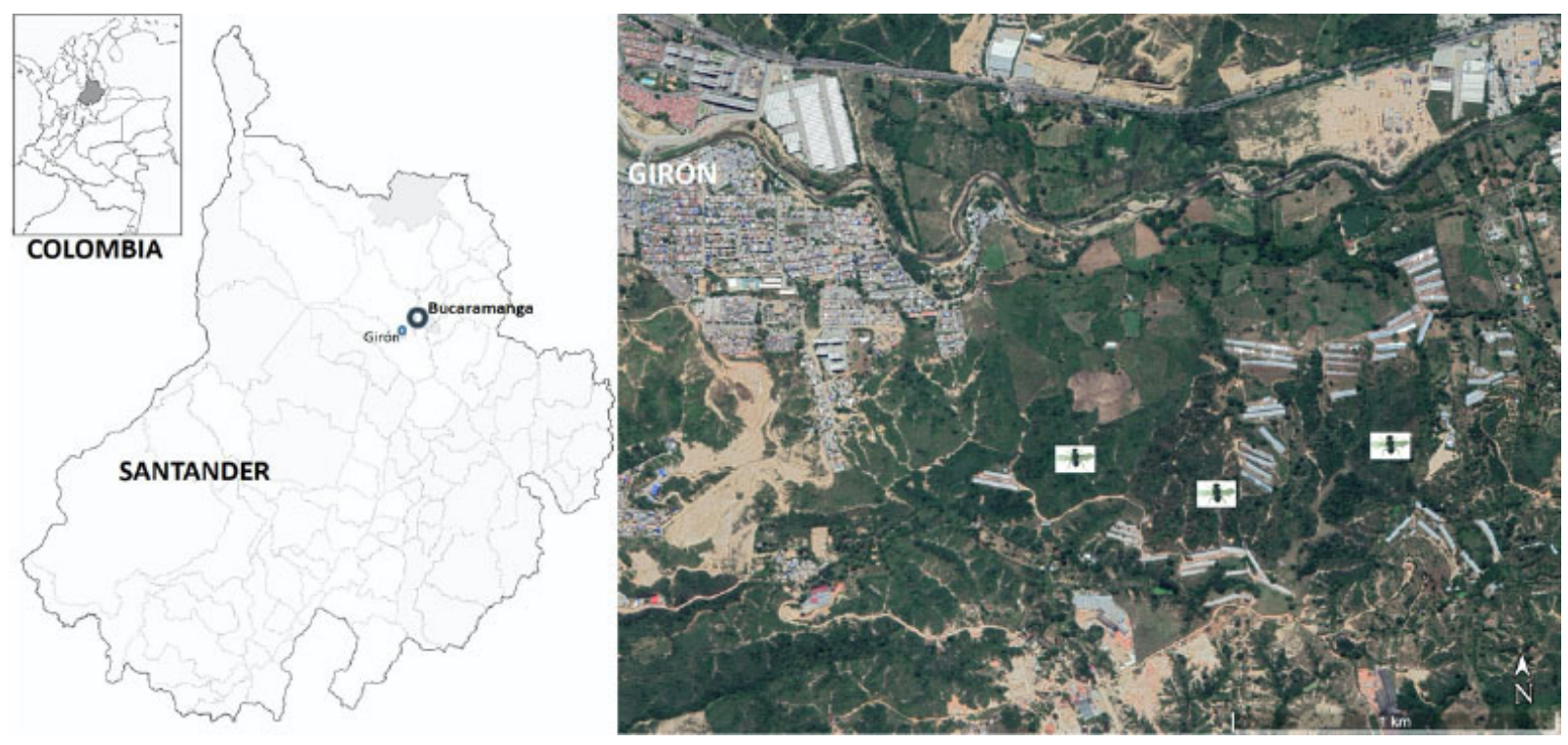

Figura 1. Localización de la fincas donde se realizaron los ensayos de evaluación de los atrayentes alimenticios con trampas McPhail para captura de M. viatrix, vereda Llanadas, Girón, Santander, Colombia (Foto tomada de GoogleEarth Pro, 2019).

Figure 1. Location of the farms where the evaluation tests of the food attractants with McPhail traps, to capture M. viatrix were carried out, Llanadas, Giron, Santander, Colombia (Photo taken from GoogleEarth Pro, 2019).

\section{Evaluación de atrayentes alimenticios en trampas McPhail}

Se evaluaron ocho atrayentes alimenticios con el propósito de determinar la efectividad para la captura de adultos de M. viatrix. Se realizó un diseño de bloques al azar con seis replicas, durante cinco semanas de muestreo. Cada tres días, las trampas se revisaron y se colocaron los atrayentes alimenticios frescos. Las trampas se instalaron a una distancia aproximada de diez metros entre ellas, y cada una era cebada con el respectivo atrayente, el cual se seleccionó al azar con fichas de papel dentro de una bolsa plástica; con esto, se aseguró la rotación de los tratamientos para compensar la heterogeneidad de las poblaciones de la mosca en campo. A los datos se les aplicó la prueba estadística ANOVA, para poder determinar diferencias en la efectividad de cada uno de los atrayentes usados. A continuación se encuentra el listado de los atrayentes alimenticios utilizados en la presente investigación, los tres primeros son los propuestos por Moreno y García (1994b) como altamente efectivos para la captura de mosca de la piña: 
- Proteína hidrolizada de soya: se diluyeron $20 \mathrm{ml}$ de proteína hidrolizada de soya por cada litro de agua.

- Vinagre y proteína hidrolizada: se mezclaron $10 \mathrm{ml}$ de vinagre de frutas y $10 \mathrm{ml}$ de proteína hidrolizada de soya por litro de agua.

- Jugo natural de piña: se licuaron durante dos minutos $500 \mathrm{~g}$ de pulpa de piña madura por litro de agua.

- Cubos de cáscara de piña: se colocaron 50 g de cáscara de piña partida en pequeños cubos dentro de cada trampa, a la cual se le agregaron $150 \mathrm{ml}$ de agua.

- Extracto de cáscara de piña: se licuaron durante dos minutos $250 \mathrm{~g}$ de cáscara de piña por litro de agua, la mezcla resultante se filtró usando un colador de tela.

- Extracto de hoja de piña: se licuaron durante dos minutos $250 \mathrm{~g}$ de hoja de piña obtenida de la corona por litro de agua, la mezcla se filtró con un colador de tela.

- Fermento con cáscara de piña: se mezclaron $500 \mathrm{~g}$ de cáscara de piña y $150 \mathrm{~g}$ de panela por litro de agua, se dejó fermentar 48 h, y se filtró con un colador de tela.

- Jugo artificial de piña: se diluyeron $20 \mathrm{~g}$ (un sobre pequeño) del producto comercial conocido como Frutiño ${ }^{\odot}$ por litro de agua.

\section{Análisis estadístico}

A pesar del amplio espectro de captura de los atrayentes evaluados, se realizaron análisis estadísticos para establecer el atrayente con mayor efectividad sobre M. viatrix, para lo cual se utilizó el programa SPSS 13.0.

Los datos de captura semanal se transformaron con $\operatorname{In}(\mathrm{x}+1)$ con el propósito de obtener la homogeneidad de varianza entre los atrayentes, así como la respectiva distribución normal (Byrkit, 1987; Guisande et al., 2006; Zar, 1999). Los datos de captura obtenidos, con los diferentes atrayentes alimenticios, fueron evaluados con un análisis de varianza (ANOVA), mediante el cual se establecieron diferencias significativas $(F=20,8775 ; p=0,000)$ en el número de individuos capturados. Una vez establecidas las diferencias significativas, se procedió a realizar una prueba a posteriori usando la prueba de Tukey. Se observó que los trozos de cáscara, el extracto de cáscara y el jugo natural de piña, presentaron los mejores resultados de captura para M. viatrix

\section{Resultados}

Un total de 138 adultos de $M$. viatrix se capturaron con los diferentes atrayentes. Los resultados de las capturas de M. viatrix con cada uno de los atrayentes evaluados en trampas McPhail, se muestran en la Figura 2, observándose que los trozos de cáscara, el extracto de cáscara y el jugo natural de piña fueron los que presentaron mayores capturas (Figura 2).

Las diferencias registradas entre las capturas semanales de M. viatrix fueron significativas para jugo natural de piña, trozos de cáscara y extracto de cáscara. Sin embargo, no se puede establecer contundentemente que alguno de ellos tuvo la efectividad suficiente como para ser implementado en programas de monitoreo y seguimiento de la especie.

\section{Discusión}

De acuerdo con los resultados obtenidos con los atrayentes alimenticios dentro de trampas McPhail, se observó que hubo poca especificidad de los atrayentes alimenticios empleados, debido a que se colectaron más de 13000 


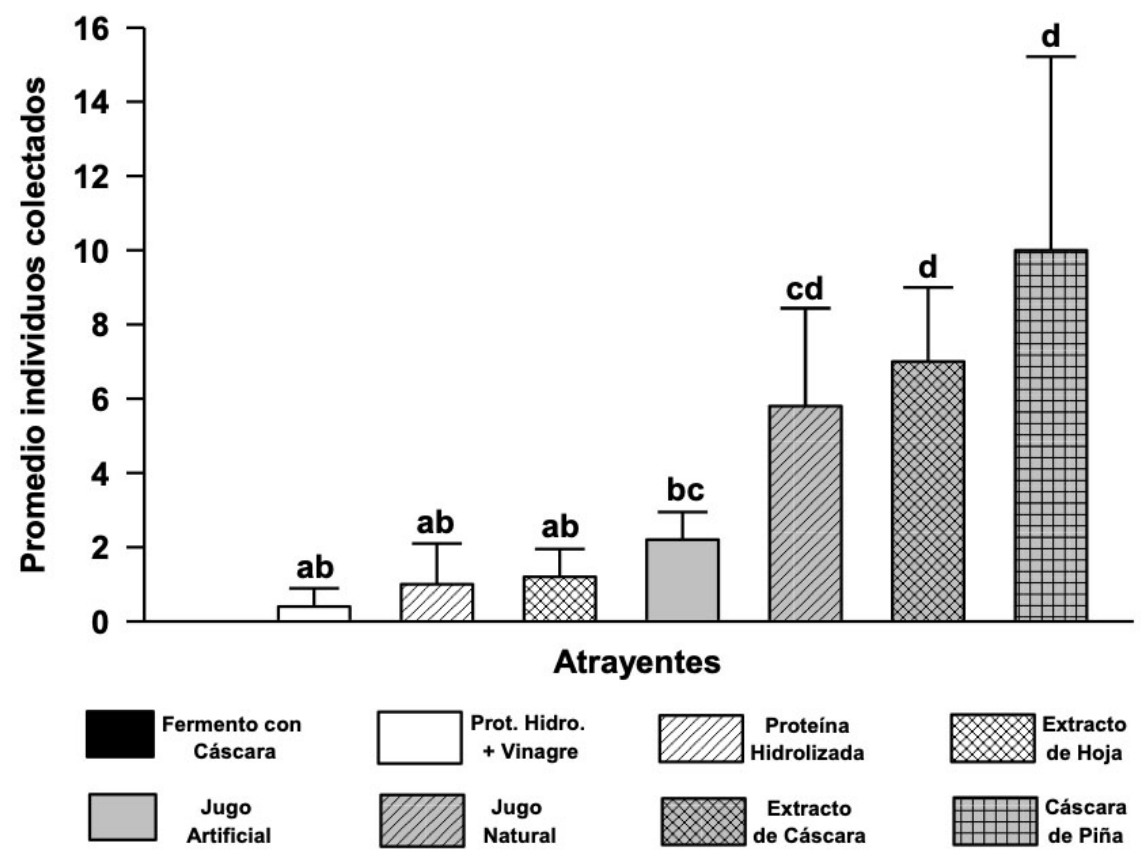

Figura 2. Prueba de Tukey para las capturas de M. viatrix con trampas McPhail, en fincas piñeras del municipio de Girón, Santander, Colombia, 2003.

Diferentes letras entre las columnas indican diferencias significativas entre los atrayentes según la prueba a posteriori de Tukey $(\mathrm{p}<0,05) . \pm$ Desviación estándar.

Figure 2. Tukey's test for the captures of M. viatrix with McPhail traps, on pineapple farms in the Giron municipality, Santander, Colombia, 2003.

Different letters between the columns indicate significant differences between the attractants according to the ex post Tukey test ( $\mathrm{p}$ $<0.05) . \pm$ Standard deviation.

individuos de diferentes órdenes, pero solo 138 adultos de M. viatrix. El manejo integrado de plagas (MIP) usado para moscas de la fruta en Colombia, y Latinoamérica en general, involucran monitoreo, aplicación de insecticidas y ciertas labores culturales, estas medidas son poco eficientes y no han cambiado en las últimas décadas (Aluja, 1994), por lo cual se hace necesario realizar evaluaciones y ajustes metodológicos para cada condición en particular.

La relativa efectividad de los trozos de cáscara de piña, el extracto de cáscara y el jugo natural de piña, especialmente los relacionados con la cáscara, puede ser explicada por las preferencias de oviposición sobre inflorescencia y fruto descritas por Arévalo y Osorio (1995). Los resultados de la presente investigación no coinciden con la eficiencia de la proteína hidrolizada que fue propuesta por Moreno y García (1994a). Es importante resaltar que con los resultados obtenidos, se tiene un buen indicio de dónde buscar las moléculas que puedan atraer eficientemente a los adultos de $M$. viatrix y ser utilizadas en planes de monitoreo.

\section{Conclusiones}

Con base en los análisis estadísticos, se observó que los trozos de cáscara, el extracto de cáscara y el jugo natural de piña, presentaron los mejores resultados de captura para M. viatrix. Sin embargo, hubo una captura de un amplio grupo de insectos, y una escasa y poco constante captura de adultos de $M$. viatrix. 
No obstante, el hecho de que dos atrayentes relacionados con cáscara de piña hayan capturado el $62,5 \%$ de todos los adultos de $M$. viatrix recolectados, se convierte en un claro indicio de que este producto podría contener moléculas o grupos de moléculas que actúen como atrayentes de dicha especie, lo cual se refuerza, adicionalmente, con el comportamiento de oviposición en campo reportado para esta especie.

\section{Agradecimientos}

El primer autor desea agradecer a sus padres y hermanos, así como a Dagoberto Arrieta Prieto, William Olarte Espinoza, Graciela Chalela Álvarez, Inés Johana Gómez y Reis. A Rubén Ariza por la idea que permitió generar esta investigación.

A los estudiantes María Isabel Puello, John James Rodríguez, Gustavo Roenes, Juliana Cuadros y Ángela Torres, por su labor en campo y laboratorio. A los cultivadores de piña por prestar sus instalaciones para las pruebas de campo y toma de muestras.

\section{Literatura citada}

Aluja, M. 1994. Bionomics and management of Anastrepha. Ann. Rev. Entomol. 39:155-178. doi:10.1146/annurev. en.39.010194.001103

Aravena, J. 2005. La expansión piñera en Costa Rica. Comunidades Ecologistas La Ceiba, San José, CRI.

Arévalo, E. 1994. Biología y hábitos del gusano de la piña Melanoloma viatrix H. Instituto Colombiano Agropecuario, Bucaramanga, COL.

Arévalo, E., y M.A. Osorio. 1995. Consideraciones generales sobre Melanoloma viatrix H. Rev. Colomb. Entomol. 21(1):1-8.

Baker, P., R.H. Herbert, and G.G. Grant. 1985. Isolation and identification of the sex pheromone of the Mediterranean fruit fly, Ceratitis capitata (Wied). J. Chem. Soc. Chem. Commun. 6:824-825. doi:10.1039/C39850000824

Bateman, M.A., and T.C. Morton. 1981. The importance of ammonia in proteinaceous attractants for fruit flies (Diptera: Tephritidae). Aust. J. Agric. Res. 32:883-903.

Bonilla, L. 1992. Cultivo de la piña. Fundación de Desarrollo Agropecuario, Santo Domingo, DOM.

Byrkit, D. 1987. Statistics today. A comprehensive introduction. Cummings Publishing Company, CA, USA.

Canal, N., M.L. Pérez, y L.F. González. 2010. La orina humana como atrayente natural de Anastrepha obliqua (Diptera: Tephritidae). Rev. Colomb. Entomol. 36(1):31-37.

Castañera, P. 2003. Control integrado de la mosca mediterránea de la fruta, Ceratitis capitata (Wiedemann) (Diptera: Tephritidae) en cítricos. Phytoma 153:131-133.

CCI (Corporación Colombia Internacional). 2006. Inteligencia de Mercados: Piña. Ministerio de agricultura y desarrollo rural, Bogotá, COL.

Chavarría, L.M. 2010. La piña. Programa Desarrollo Económico Sostenible en Centroamérica, Tegucigalpa, HON.

FAO. 2012. FAOSTAT: Food and agriculture data. FAO, Rome, ITA. http://faostat.fao.org/ (accessed Jul. 14, 2019).

Galindo, J.R. 1994a. Fusariosis (Fusarium moniliforme Sheld) en piña. Instituto Colombiano Agropecuario, Bucaramanga, COL. 
Galindo, J.R. 1994b. Resistencia de microorganismos al uso de pesticidas. Instituto Colombiano Agropecuario, Bucaramanga, COL.

Gallego, F.L. y R. Vélez. 1992. Lista de insectos que afectan los principales cultivos, plantas forestales, animales domésticos y al hombre en Colombia. Medellín, Colombia. Universidad Nacional de Colombia, COL.

García, A., y J.A. Martínez. 1991. Manejo Integrado del cultivo de la piña en Santander. Instituto Colombiano Agropecuario, Bucaramanga, COL.

Garita, R.A. 2014. La Piña. Editorial Tecnológica de Costa Rica,Cartago, CRI.

Guisande, C., A. Barreiro, I. Maneiro, I. Riveiro, A.R. Vergara, y A. Vaamonde. 2006. Tratamiento de datos. Ediciones Diaz de Santos, Madrid, ESP.

Gutiérrez, J.M., G. Martínez, A. Villaseñor, W. Enkerlin, y F. Hernández. 2013. Los programas de moscas de la fruta en México. Su historia reciente. Instituto Interamericano de Cooperación para la Agricultura, México, D.F., MEX.

Heath, R.R., N.D. Epsky, D. Midgarden, and B.I. Katsoyannos. 2004. Efficacy of 1,4-diaminobutane (putrescine) in a foodbased synthetic attractant for capture of Mediterranean and Mexican fruit flies (Diptera: Tephritidae). J. Econ. Entomol. 97:1126-1131. doi:10.1603/0022-0493(2004)097[1126:eodpia]2.0.co;2

Hendel, F. 1911. Die arten der Dipteren-Subfamilie Richardiinae. Deutsch. Ent. Zeitschr. 2:181-396.

Higuera, C. 1994. El cultivo de la piña en Santander. Instituto Colombiano Agropecuario, Bucaramanga, COL.

ICA (Instituto Colombiano Agropecuario). 1991. Nueva plaga. Notas y noticias entomológicas. ICA, Bogotá, COL.

ICA (Instituto Colombiano Agropecuario). 2011. Manual técnico de trampeo de moscas de la fruta. ICA, Bogotá, COL.

Jensen, W., y F.B. Salisbury. 1988. Botánica. 2da ed. McGraw-Hill, México, D.F., MEX.

Katsoyannos, B.I., R.R. Heath, N.T. Papadopoulos, N.D. Epsky, and J. Hendrichs. 1999. Field evaluation of Mediterranean fruit fly (Diptera: Tephritidae) female selective attractants for use in monitoring programs. J. Econ. Entomol. 92:583-589. doi: $10.1093 /$ jee/92.3.583

La República. 2012. Producción de piña es significativa en el país. La República, COL. https://www.larepublica.co/archivo/ produccion-de-pina-es-significativa-en-el-pais-2018079 (consultado 17 abr. 2015).

León, G. 1988. Fluctuación poblacional y manejo de Anastrepha spp. en mango y guayaba. En: A. Bustillo, editor, Seminario sobre moscas de las frutas. Seminarios Sociedad Colombiana de Entomología, Medellín, COL.

López, D. 1988. Detección de adultos de moscas de las frutas. En: A. Bustillo, editor, Seminario sobre moscas de las frutas. Seminarios Sociedad Colombiana de Entomología, Medellín, COL.

López, D. 1989. Detección de adultos de moscas de las frutas por medio de trampas. En: D.F. López y L. Núñez, editores, Curso sobre moscas de la fruta. Instituto Colombiano Agropecuario, La Ceja, COL.

Martínez, J.A. 1994. Acciones ejecutadas por el ICA para controlar el gusano de la piña en Santander. En: P. Martínez et al., editores, Curso manejo integrado del cultivo de la piña. Instituto Colombiano Agropecuario, Bucaramanga, COL.

Matheus, H. 2005. Las moscas de la fruta. ICA, Bogotá, COL.

Mazor, M., S. Gothilf, and R. Galun. 1987. The role of ammonia in the attraction of females of the Mediterranean fruit fly to protein hydrolysate baits. Entomol. Experiment. Applicata 43:25-29. doi:10.1111/j.1570-7458.1987.tb02198.x

Montilla, R., J.L. García, L. LaCruz, y D. Durán. 2007. Spalangia drosophilae Ashmead (Hymenoptera: Pteromalidae) parasitoide de pupas de la mosca de la piña Melanoloma viatrix Hendel (Diptera: Richardiidae) en Trujillo, Venezuela. Agron. trop. 57(2): 107-112. 
Moreno, P., y A. García. 1994a. Evaluación de cebos tóxicos en el manejo de Melanoloma viatrix. En: P. Martínez et al., editores, Curso de manejo integrado del cultivo de la piña. Instituto Colombiano de Agricultura, Bucaramanga, COL.

Moreno, F.A., y A. García. 1994b. Evaluación de atrayentes de Melanoloma viatrix. En: P. Martínez et al., editores, Curso de manejo integrado del cultivo de la piña. Instituto Colombiano Agropecuario, Bucaramanga, COL.

OIEA (Organismo Internacional de Energía Atómica). 2005. Guía para el trampeo en programas de control de la mosca de la fruta en áreas amplias. OIEA, Viena, AUS.

Papadopoulos, N.T., B.I. Katsoyannos, N.A. Kouloussis, J. Hendrichs, J.R. Carey, and R.R. Heath. 2001. Early detection and population monitoring of Ceratitis capitata (Diptera: Tephritidae) in a mixed-fruit orchard in northern Greece. J. Econ. Entomol. 94:971-978. doi:10.1603/0022-0493-94.4.971.

Pacheco, N.D. 2016. Comparación de las respuestas de Anastrepha ludens y Anastrepha obliqua (Díptera: Tephritidae) al atrayente sintético "BioLure". El Colegio de la Frontera Sur, Chiapas, MEX.

Piñero, J., M. Aluja, A. Vázquez, M. Equihua, and J. Varón. 2003. Human urine and chicken feces as fruit fly (Diptera: Tephritidae) attractants for resource-poor fruit growers. J. Econ. Entomol. 96:334-339. doi:10.1603/0022-0493-96.2.334

Primo-Millo, E., F. Alfaro-Lassala, y R. Argilés-Herrero. 2003. Plan de actuación contra la mosca de las frutas (Ceratitis capitata) en la comunidad Valenciana. Phytoma 153:127-130.

Raga, D., and M.E. Sato. 2005. Effect of spinosad bait against Ceratitis capitata (Wied.) Anastrepha fraterculus (Wied.) (Diptera: Tephritidae) in laboratory. Neotrop. Entomol. 43:815-822. doi:10.1590/S1519-566X2005000500014

Rodriguez, G.G., P.D. Mark, R. Silva-Acuña, E. Gonzalez, y E. Milano. 2000. Evaluación de trampas y formulaciones atrayentes para la captura de la mosca de la guayaba, Anastrepha striata Schiner (Diptera: Tephritidae) en Santa Bárbara, Monagas, Venezuela. Bol. Entomol. Venezolana 15(1):49-60.

Salazar, R. 1994. Situación del cultivo de la piña en Colombia. En: P. Martínez et al., editores, Curso de manejo integrado del cultivo de la piña. Instituto Colombiano Agropecuario, Bucaramanga, COL.

Salles, L.A. 1997. Sucos de frutas como atractivos para a captura de adultos da moscas-das-frutas Anastrepha fraterculus (Wied. 1930) (Diptera: Tephritidae). Pesq. Agropecu. Gaúcha 3(1):25-28.

Sandoval, I.A., y E.E. Torres. 2011. Guía técnica del cultivo de la piña. Centro nacional de tecnología agropecuaria y forestal "Enrique Álvarez Córdova", San Andrés, ESA.

Santoyo, J.A., y C.O. Martínez. 2011. Paquete tecnológico para la producción de piña en el sur de Sinaloa. Fundación Produce Sinaloa, Rosario, MEX.

Veloso, V.R.S., P.M. Fernandes, M.R. Rocha, M.V. Queiroz, e R.M.R. Silva. 1994. Armadilha para o monitoramento e controle das moscas-das-frutas Anastrepha spp. e Ceratitis capitata (Wied.). Anais Soc. Entomol. Bras. 23:487-493.

Villalobos-Moreno, A., J.C. Agudelo, A.F. Barajas, S.G. Herrera, y J.C. Céspedes. 2009. Cambios morfológicos durante la ontogenia pupal de Melanoloma viatrix (Diptera: Richardiidae). Rev. Colomb. Entomol. 35(1):98-100.

Villalobos-Moreno, A., y J.E. Luque. 2012. Aspectos biológicos de Melanoloma viatrix Hendel, 1911 (Diptera: Richardiidae) en condiciones de laboratorio. Rev. Agric. Trop. 35(5-6):52-67.

Villalobos-Moreno, A., J.E. Luque, y J. Villamizar. 2013. Parasitoides de mosca de la piña Melanoloma viatrix Hendel, 1911 (Diptera: Richardiidae) en tres regiones piñeras de Santander. Rev. Agric. Trop. 36(3-4):29-36.

Zar, H. 1999. Biostatistical Analysis. Prentice Hall, NJ, USA. 